TITLE:

\title{
Enhancing recombinant protein production in human cell lines with a constitutive transport element and mRNA export proteins.
}

\section{$\operatorname{AUTHOR}(\mathrm{S}):$}

Aihara, Yuki; Fujiwara, Naoko; Yamazaki, Tomohiro; Kambe, Taiho; Nagao, Masaya; Hirose, Yutaka; Masuda, Seiji

\section{CITATION:}

Aihara, Yuki ... [et al]. Enhancing recombinant protein production in human cell lines with a constitutive transport element and mRNA export proteins.. Journal of biotechnology 2011, 153(04-3): 86-91

\section{ISSUE DATE:}

2011-05-20

URL:

http://hdl.handle.net/2433/141844

\section{RIGHT:}

@ 2011 Elsevier B.V.; この論文は出版社版でありません。引用の際には 出版社版をご確認ご利用ください。; This is not the published version. Please cite only the published version. 
Enhancing recombinant protein production in human cell lines with a constitutive transport element and mRNA export proteins

Yuki Aihara ${ }^{a}$, Naoko Fujiwara ${ }^{a}$, Tomohiro Yamazaki ${ }^{a}$ Taiho Kambe ${ }^{a}$, Masaya Nagao, Yutaka Hirose $^{\mathrm{b}}$ and Seiji Masuda

a'Division of Integrated Life Science, Graduate School of Biostudies, Kyoto University, Kyoto, Japan.

${ }^{\mathrm{b}}$ Graduate School of Medicine and Pharmaceutical Sciences, University of Toyama, Toyama, Japan.

Corresponding Author: Seiji Masuda

Division of Integrated Life Science

Graduate School of Biostudies, Kyoto University

Kyoto 606-8502, Japan.

Tel: 81-75-753-9430

Fax: 81-75-753-6274

E-mail: masuda@kais.kyoto-u.ac.jp

\section{Abbreviations}

CMV, cytomegalovirus; CTE, constitutive transport element; EPO, erythropoietin; RSV, Rous sarcoma virus; SEAP, secreted alkaline phosphatase; SV40, Simian virus 40. 


\begin{abstract}
Recent research into mRNA maturation processes in the nucleus has identified a number of proteins involved in mRNA transcription, capping, splicing, end processing and export. Among them, the Tap-p15 heterodimer acts as an mRNA export receptor. Tap-p15 is recruited onto fully processed mRNA in the nucleus, which is ready for export to the cytoplasm, through associating with Aly or SR proteins on mRNA, or by directly associating with a constitutive transport element (CTE), an RNA element derived from type D retroviruses. mRNA containing a CTE is exported to the cytoplasm by directly associating with Tap-p15, even in the absence of Tap-recruiting proteins such as Aly or SR proteins on the mRNA. Here, we showed that the use of a CTE enhanced the expression of recombinant protein in human cell lines. The co-expression of reporter proteins and Tap-p15 also enhanced recombinant protein expression. Moreover, the use of a CTE and Tap-p15 synergistically further enhanced the recombinant protein expression. In addition to Tap-p15, several Tap-p15-recruiting proteins, including Aly and SR proteins, enhanced recombinant protein expression, albeit independently of the CTE. The incorporation of a CTE and Tap-p15-recruiting proteins into protein expression system is useful to increase recombinant protein yield in human cells.
\end{abstract}

\title{
Key words
}

constitutive transport element, mRNA export, Tap-p15, mRNA processing factor 


\section{Introduction}

To express recombinant proteins in animal cells, expression cassettes have been developed, which contain a promoter, a multicloning site to insert the gene of interest and a polyadenylation signal in a plasmid. Simian virus 40 (SV40) and Rous sarcoma virus (RSV) promoters were used in the early stages of recombinant protein expression (Das et al., 1985; Laimins et al., 1984; Yamamoto et al., 1980), but these promoters have disadvantages. The SV40 promoter is relatively weak and the RSV promoter is cell-type specific. The search for strong, ubiquitous promoters identified the cytomegalovirus (CMV) promoter and the eukaryotic initiation factor 1 alpha promoter (Mizushima and Nagata, 1990; Stenberg et al., 1984; Stinski and Roehr, 1985; Wakabayashi-Ito and Nagata, 1994). At present, these promoters are the most frequently used, both for research and industrial recombinant protein production.

In eukaryotic cells, protein-coding genes are first transcribed as a pre-mRNA in the nucleus. The pre-mRNA undergoes several RNA processing steps, such as 5'-capping, splicing and 3'-end processing. The mature mRNA is exported from the nucleus to the cytosol through the nuclear pore complex and is translated. These gene expression processes are tightly coordinated with each other, to achieve efficient and accurate gene expression (Komili and Silver, 2008; Maniatis and Reed, 2002;

Orphanides and Reinberg, 2002). In addition to the mRNA processing steps, the nuclear export of mRNA is essential for eukaryotic gene expression. Studies of the mRNA export pathway led to the identification of the most important factors (Aguilera, 2005; Kohler and Hurt, 2007; Reed and Hurt, 2002; Vinciguerra and Stutz, 2004); these being the hTREX complex and several SR proteins (Cheng et al., 2006; Huang et al., 2003; Huang et al., 2004; Jimeno et al., 2002; Katahira et al., 2009; Masuda et al., 2005; Reed and Cheng, 2005; Strasser et al., 2002). When mRNA maturation is completed, mRNA 
is recognized by a heterodimer of Tap (also called NXF1) and p15 (also called NXT1), an mRNA export receptor, through associating with the adaptor protein, Aly (a component of the hTREX complex) or SR proteins, including 9G8 and SF2, and is exported to the cytoplasm (Cullen, 2003; Katahira et al., 1999; Stutz and Izaurralde, 2003). In the absence of the Tap-p15 association, the mRNA is retained in the nucleus by the mRNA quality control system (Fasken and Corbett, 2009; Moraes). Thus, the Tap-p15 association with mRNA is an essential step to export most mRNAs, and it functions to integrate several mRNA export pathways. If the expression of Tap or $\mathrm{p} 15$ is the limiting step for mRNA export, the forced expression of Tap or p15 may affect protein expression. In the same manner, when the requirement for Tap or p15 is the limiting step for mRNA export, the overexpression of these proteins may efficiently recruit Tap-p15 onto mRNA.

Type D retroviruses such as simian retrovirus type 1 , simian retrovirus type 2 and Mason-Pfizer monkey virus contain a conserved cis-acting constitutive transport element (CTE) (Bray et al., 1994; Tabernero et al., 1996; Zolotukhin et al., 1994) that is necessary for virus replication. Tap directly binds to the CTE without any associated proteins and mediates nuclear export of CTE-containing RNAs even if the mRNA contains introns (Bear et al., 1999; Braun et al., 1999; Fontoura et al., 2005; Zolotukhin et al., 2001). CTE-containing mRNA can, therefore, bypass the mRNA quality control system. The interaction between Tap and the CTE, and the processes of viral protein production, have been intensively studied, because some viral protein expression is tightly restricted by the CTE. However, CTEs and Tap have not been used for recombinant protein expression in general.

Here, we attempted to improve current methods for recombinant protein production in human cells using mRNA processing factors and a CTE. These elements 
can be incorporated into pre-existing expression systems, including in the basic research for the protein expression to detect protein-protein interaction, the purification of protein complex and the protein-nucleic acid interaction, and the industrial recombinant protein production. We provide evidence that overexpression of Tap-p15, Tap-p15-recruiting proteins and a CTE independently and synergistically support protein production. These findings provide new insight into cellular engineering for gene expression regulated by the mRNA export machinery.

\section{Material and Methods}

\subsection{Plasmid construction}

To construct pGL3LUC-CTE, the CTE fragment was released from pRSVLUC-CTE by Xba I and was ligated into pGL3LUC. Full-length secreted alkaline phosphatase (SEAP) and erythropoietin (EPO) fragments were obtained by PCR amplification with the addition of restriction enzyme sites at both ends. pcDNA5SEAP and pcDNA5EPO were constructed by subcloning full-length SEAP and EPO containing Bam $\mathrm{HI}$ and Xho I sites at their ends into the Bam $\mathrm{HI}$ and Xho I sites, respectively, of pcDNA5. pcDNA5SEAP-CTE and pcDNA5EPO-CTE were constructed by subcloning a CTE into the Xho I site of pcDNA5SEAP and pcDNA5EPO, respectively. pCMVFlagp15 was constructed by subcloning full-length p15 containing Eco RI and Xho I sites at its ends into the Eco RI and Xho I sites, respectively, of pCMV2B. pcDNA5Flag9G8 and pcDNA5FlagSF2 were constructed by subcloning full-length 9G8 and SF2 into the Bam HI and Xho I sites of pcDNA5Flag. pcDNA5FlagAly was constructed by subcloning full-length Aly into the Bam $\mathrm{HI}$ and Eco RV sites of pcDNA5Flag. The correct construction of each plasmid was confirmed by sequencing. 


\subsection{Cell culture and transfection}

All cell lines used in this study were maintained in Dulbecco's modified Eagle's medium (Wako, Osaka, Japan) supplemented with $10 \%$ heat-inactivated fetal bovine serum under normoxic conditions $\left(5 \% \mathrm{CO}_{2}, 21 \% \mathrm{O}_{2}\right.$ and $\left.74 \% \mathrm{~N}_{2}\right)$ in a humidified $\mathrm{CO}_{2}$ incubator at $37^{\circ} \mathrm{C}$. Cells $\left(4 \times 10^{4}\right)$ were inoculated in a 24 -well plate and cultured for $24 \mathrm{~h}$. Transient transfection of CTE-containing plasmids $(0.04 \mu \mathrm{g})$ was performed with p $\beta$ act $\beta g a l(0.16 \mu \mathrm{g})$ using Lipofectamine 2000 (Invitrogen,

Carlsbad,CA) reagent according to the manufacturer's instructions. Co-transfection of CTE-containing plasmids $(0.04 \mu \mathrm{g})$ and RNA binding proteins such as Tap (0.08 $\mu \mathrm{g})$ and p15 $(0.08 \mu \mathrm{g})$ was performed using Lipofectamine 2000 reagent.

\subsection{Establishment of EPO-producing cell lines}

Flpin293Trex-EPO and Flpin293Trex-EPO-CTE cell lines were obtained by co-transfection of pcDNA5EPO or pcDNA5EPO-CTE, and pOG44 into Flpin293Trex cells. The colonies were selected with $100 \mu \mathrm{g} / \mathrm{ml}$ hygromycin for 10 days. EPO-expressing clones were selected by the EPO assay described below. EPO expression was induced by the addition of doxycycline $(0.5 \mu \mathrm{g} / \mathrm{ml})$ for $48 \mathrm{~h}$. Transient expression of mRNA binding proteins was performed using expression plasmids $(0.2$ $\mu \mathrm{g})$ and Lipofectamine 2000 reagent, according to the manufacturer's instructions. After $4 \mathrm{~h}$ of transfection, EPO expression was induced by the addition of doxycycline $(0.5$ $\mu \mathrm{g} / \mathrm{ml})$ for $48 \mathrm{~h}$.

\subsection{Reporter assay and western analysis}

The culture supernatant and cell lysate were recovered after $24 \mathrm{~h}$ of 
transfection unless otherwise indicated. The cells were washed with phosphate buffered saline and lysed with passive lysis buffer (Promega, Madison, WI) for $30 \mathrm{~min}$. The clear lysate obtained by a brief spin was used to measure the luciferase activity, $\beta$-galactosidase activity and the total protein content. The supernatant was collected and the SEAP and EPO productions were measured.

Luciferase activity was measured as described previously (Fujiwara et al., 2010). SEAP activity in the culture supernatant was measured as described previously (Suzuki et al., 2005). EPO activity in the culture supernatant was measured by a sandwich enzyme-linked immunosorbent assay as described previously (Masuda et al., 2000a; Masuda et al., 2000b; Masuda et al., 1999). $\beta$-galactosidase activity was measured as described previously (Masuda et al., 2000b). The total protein content was measured using a protein assay kit (Nacalai, Kyoto, Japan).

The EPO protein production during the long-term culture was examined as described previously (Nagao et al., 1992). Briefly, the cells were cultured confluent with Dulbecco's modified Eagle's medium supplemented with $10 \%$ heat-inactivated fetal bovine serum in a $9 \mathrm{~cm}$ tissue culture dish. The media was exchanged to $6 \mathrm{ml}$ OPTI-MEM (Invitrogen) supplemented with $0.5 \%$ heat-inactivated fetal bovine serum. The media was replaced to a fresh media at 24 hours interval. To detect EPO in the culture supernatant by western analysis, the supernatant was concentrated ten times by the centrifuge enrichment. The concentrated sample was separated by sodium dodecyl sulfate-polyacrylamide gel electrophoresis. EPO was detected with rabbit anti-EPO polyclonal antibody (Masuda et al., 1994; Okano et al., 1993). Proteins were separated by sodium dodecyl sulfate-polyacrylamide gel electrophoresis. Western analysis was performed as described previously (Yamazaki et al., 2010). For western blotting, anti-Flag (M2; Sigma, St. Louis, MO) and HA (12CA5; Sigma) antibodies were used as 
primary antibodies to detect Flag- and HA-tagged proteins, respectively. Rabbit anti-EPO antibody (Masuda et al., 1994) was used to detect EPO protein.

\section{Results}

The processes of gene expression in mammalian cells was shown in Supplemental Figure 1A. To evaluate the effect of CTEs on protein expression in general, we inserted a CTE into several reporter constructs with different combinations of promoters (SV40 or CMV) and reporter proteins (luciferase, SEAP or EPO) (Supplemental Figure 1B). Luciferase is a very commonly used reporter protein. SEAP is also frequently used as a reporter; it consists of a homodimer and is secreted into the culture media, enabling assay of the culture media to quantify recombinant protein production. EPO is a heavily glycosylated protein that is also secreted into the culture media. It is used for the pharmaceutical products.

We first examined the expression of luciferase in several human cell lines. The transfection efficiency was normalized to $\beta$-galactosidase. The presence of the CTE increased the expression of luciferase 1.4-2.4-fold in all four human cell lines examined, HeLa, MCF7, U2OS and A549 (Figure 1).

To evaluate the effect of Tap-p15 in CTE-containing expression vectors, Tap-p15 was co-expressed in HeLa cells with or without a CTE. Tap-p15 expression enhanced the expression of the luciferase, SEAP and EPO reporters even in the absence of the CTE (Figure 2A, C, E, respectively). The expression of Tap and p15 was confirmed by western blotting (Figure 2B, D, F). In the presence of the CTE, Tap-p15 expression enhanced luciferase, SEAP and EPO expression still further (Figure 2A, C, E, respectively). These results indicate that Tap-p15 enhances gene expression, probably by promoting mRNA export, and that these effects are increased in the 
presence of the CTE.

Although the CTE and Tap-p15 increased transient recombinant protein production in human cell lines, precise comparisons could not be made because the overexpression of Tap-p15 per se enhanced gene expression in general, including that of $\beta$-galactosidase, and therefore the normalization was performed by total protein content in the transient transfection. To overcome this issue, we established the stably expressing cell lines Flpin293Trex-EPO and Flpin293Trex-EPO-CTE. In Flpin293Trex cell lines, an exogenous plasmid can be integrated into a single defined genomic locus and the expression level of the recombinant protein is extremely similar among clones (Sauer, 1994). In other words, the effect of integration site on gene expression and the copy number of integrated genes need not be considered. We confirmed that EPO secretion into the media was the same in three independent Flpin293Trex-EPO lines (data not shown). Using this expression system, we compared EPO production with or without the CTE. In the presence of the CTE, EPO production was enhanced approximately two-fold (Figure 3A). The EPO proteins produced from both cell lines were highly similar (Figure 3B). Next, the EPO productivity during the long-termination was measured by a sandwich enzyme-linked immunosorbent assay. The EPO production was approximately $3 \mu \mathrm{g} / 10^{6} / \mathrm{day}$ ( $4 \mathrm{mg} / \mathrm{L} /$ day) without CTE element. In contrast, the EPO production was enhanced to 5-6 $\mu \mathrm{g} / 10^{6} / \mathrm{day}$ (7-8 $\mathrm{mg} / \mathrm{L} /$ day) during the culture period. These results suggest that the CTE is also functional in stably expressing cell lines.

To estimate the effect of Tap-p15 and Tap-p15 recruiting proteins, Tap-p15, 9G8, SF2 or Aly were transiently transfected into 293Trex-EPO cells (Figure 4A). Expression of these proteins was confirmed by western blotting (Figure 4B). The overexpression of Tap-p15 and Tap-p15-recruiting proteins enhanced EPO production. 
In Flpin293Trex-EPO-CTE cells, the transient expression of Tap-p15 and Tap-p15-recruiting proteins further enhanced EPO production (Figure 4C). These results clearly indicate that the overexpression of mRNA processing factors positively affects recombinant EPO protein production in the presence or absence of a CTE.

We also evaluated the effect of the CTE in the widely used animal cell lines $\mathrm{CHO}$ and $\mathrm{BHK}$, to assess its applicability in improving pharmaceutical protein production. Surprisingly, protein expression was decreased in both cell lines when the CTE was incorporated (Supplemental Figure 2A and B). Co-expression with human Tap-p15 recovered the protein expression to the levels without the CTE, suggesting that human Tap is required for CTE-containing expression systems, and that hamster Tap might not associate with the CTE (Supplemental Figure 2C). This result indicates that CTE is useful for the recombinant protein expression in human cell lines.

\section{Discussion}

The CTE is an essential element for the replication of several viruses (Bray et al., 1994; Ernst et al., 1997; Tabernero et al., 1996; Zolotukhin et al., 1994). The CTE functions to export CTE-containing RNA by directly interacting with the mRNA export receptor Tap. Here, we examined the effect of the CTE on recombinant protein expression in human cell lines. We showed that the CTE promoted the expression of the three different transiently transfected reporter proteins and the one stably transfected reporter protein tested. This indicates that the CTE is useful for enhancing recombinant protein expression in human cell lines.

The overexpression of Tap-p15 promoted recombinant protein production independent of the CTE, indicating that normal levels of Tap in these cells are insufficient for maximum production of recombinant protein. Similarly, the 
overexpression of Tap-p15-recruiting proteins, 9G8, SF2 and Aly, resulted in enhanced production of recombinant protein. Aly is a component of the hTREX complex and functions to recruit Tap onto mRNA (Zhou et al., 2000). 9G8 and SF2 also function to recruit Tap onto mRNA (Huang et al., 2003; Huang et al., 2004); this common function is probably the role played by these proteins in recombinant protein expression. The fold induction of EPO protein by Tap-p15 was not as effective as that induced by 9G8 expression. There are at least two explanations for this. One is that the expression level of exogenous Tap-p15 was much less than that of 9G8 (Figure 5B and D) and therefore the effect of Tap-p15 could not reach its maximum level. Another explanation is that 9G8 expression is the limiting step for mRNA export in Flpin293Trex cells. Overall however, the expression of Tap-p15 and Tap-p15-recruiting proteins regulates the levels of recombinant protein expression. Further determination of the levels of protein in each cell line will assist the optimization of recombinant protein expression.

For basic research, the incorporation of CTE element into pre-existing expression cassette will further enhance the protein expression of interest. Our approach may support the biochemical and biological analysis using human cells with low efficiency of transfection. For industrial and pharmaceutical protein production, most therapeutic recombinant proteins are now produced in Chinese hamster ovary $(\mathrm{CHO})$ cells, because they have been extensively characterized (Durocher and Butler, 2009; Li and d'Anjou, 2009; Mohan et al., 2008; Sethuraman and Stadheim, 2006). Other animal cell lines, including three of human origin, are also used for therapeutic recombinant protein production, for example, BHK, HT1080, Namalwa and HEK293 (Durocher and Butler, 2009; Li and d'Anjou, 2009). One advantage of human cells is that human cell-based expression systems are expected to produce recombinant proteins with post-translational modifications more similar to their natural counterparts. There are at 
least three post-translational differences between human cells and, $\mathrm{CHO}$ and $\mathrm{BHK}$ cells. First, BHK and $\mathrm{CHO}$ cells lack certain sugar-transferring enzymes (e.g., alpha1-3/4 fucosyltransferases, alpha2-6 sialyltransferases) (Grabenhorst et al., 1999). As a result, these cells do not synthesize particular human tissue-specific terminal carbohydrate motifs required for its biological activity (Lee et al., 2009; Seppala et al., 2007). Second, gamma-carboxyglutamyl residues are essential for some proteins, such as protein $\mathrm{C}$ (Suttie, 1986). Third, the potentially immunogenic N-glycolylneuraminic acid residues are synthesized in most mammalian cells, including $\mathrm{CHO}$ and $\mathrm{BHK}$ cells, but are not synthesized in human cells (Padler-Karavani et al., 2008; Tangvoranuntakul et al., 2003). Therefore, production of therapeutic proteins in human cells is also expected to reduce potential immune reactions against non-human epitopes. Thus, research into improving yields of protein production in human cells is highly worthwhile.

In the present study, we used the human cell line to examine the effect of CTE and Tap-p15-recruiting proteins whether the use of CTE enhanced the recombinant protein productivity. The enhancement of EPO productivity is stable and 1.7 to 2 fold during the culture periods, indicating this production system is useful for the recombinant protein production. The productivity of EPO protein was less than 10 $\mu \mathrm{g} / 10^{6} /$ day because Flpin293Trex-EPO and Flpin293Trex-EPO-CTE cell lines contained just one transgene in the defined genome locus. Therefore Flpin293Trex cell line was not adequate for the summit production of recombinant protein but suitable to compare the recombinant production on the same background. Recent mammalian cells have high productivity of $20-60 \mu \mathrm{g} / 10^{6} /$ day although most of these results were obtained from CHO cells (Demain and Vaishnav, 2009). The incorporation of multi-copy transgene into active genome locus of the parental HEK293 cell line will further enhance the EPO production. At present, the use of a CTE in recombinant 
protein production is advantageous in human cells, but not in $\mathrm{CHO}$ and $\mathrm{BHK}$ cells. To search for a CTE-like element that directly recruits hamster Tap onto mRNA might be a promising strategy for the enhancement of recombinant protein production in $\mathrm{CHO}$ and BHK cells.

\section{Acknowledgments}

We thank Dr. T. Kimura and Dr. B.K. Ferber for kindly providing pRSVLUC-CTE and pCMVHATap, respectively. This work was supported by grants-in-aid from the Ministry of Education, Culture, Sports, Science and Technology of Japan, JST in Research for Promoting Technological Seeds, the Noda Institute for Scientific Research, the Suzuken Memorial Foundation and the Asahi Glass Foundation (to S. M.).

\section{References}

Aguilera, A., (2005) Cotranscriptional mRNP assembly: from the DNA to the nuclear pore. Curr Opin Cell Biol 17, 242-250.

Bear, J., Tan, W., Zolotukhin, A.S., Tabernero, C., Hudson, E.A., Felber, B.K., (1999) Identification of novel import and export signals of human TAP, the protein that binds to the constitutive transport element of the type D retrovirus mRNAs. Mol Cell Biol 19, 6306-6317.

Braun, I.C., Rohrbach, E., Schmitt, C., Izaurralde, E., (1999) TAP binds to the constitutive transport element (CTE) through a novel RNA-binding motif that is sufficient to promote CTE-dependent RNA export from the nucleus. Embo J 18, 
1953-1965.

Bray, M., Prasad, S., Dubay, J.W., Hunter, E., Jeang, K.T., Rekosh, D., Hammarskjold, M.L., (1994) A small element from the Mason-Pfizer monkey virus genome makes human immunodeficiency virus type 1 expression and replication Rev-independent. Proc Natl Acad Sci U S A 91, 1256-1260.

Cheng, H., Dufu, K., Lee, C.S., Hsu, J.L., Dias, A., Reed, R., (2006) Human mRNA export machinery recruited to the 5' end of mRNA. Cell 127, 1389-1400.

Cullen, B.R., (2003) Nuclear RNA export. J Cell Sci 116, 587-597.

Das, G.C., Niyogi, S.K., Salzman, N.P., (1985) SV40 promoters and their regulation. Prog Nucleic Acid Res Mol Biol 32, 217-236.

Demain, A.L., Vaishnav, P., (2009) Production of recombinant proteins by microbes and higher organisms. Biotechnol Adv 27, 297-306.

Durocher, Y., Butler, M., (2009) Expression systems for therapeutic glycoprotein production. Curr Opin Biotechnol 20, 700-707.

Ernst, R.K., Bray, M., Rekosh, D., Hammarskjold, M.L., (1997) Secondary structure and mutational analysis of the Mason-Pfizer monkey virus RNA constitutive transport element. Rna 3, 210-222. 
Fasken, M.B., Corbett, A.H., (2009) Mechanisms of nuclear mRNA quality control. RNA Biol 6, 237-241.

Fontoura, B.M., Faria, P.A., Nussenzveig, D.R., (2005) Viral interactions with the nuclear transport machinery: discovering and disrupting pathways. IUBMB Life 57, $65-72$.

Fujiwara, N., Yoshikawa, M., Yamazaki, T., Kambe, T., Nagao, M., Masuda, S., (2010) A screening method tuned for mRNA processing factors in human cells by evaluation of the luciferase reporter activity and the subcellular distribution of bulk poly(A)+ RNA. Biosci Biotechnol Biochem 74, 1512-1516.

Grabenhorst, E., Schlenke, P., Pohl, S., Nimtz, M., Conradt, H.S., (1999) Genetic engineering of recombinant glycoproteins and the glycosylation pathway in mammalian host cells. Glycoconj J 16, 81-97.

Huang, Y., Gattoni, R., Stevenin, J., Steitz, J.A., (2003) SR splicing factors serve as adapter proteins for TAP-dependent mRNA export. Mol Cell 11, 837-843.

Huang, Y., Yario, T.A., Steitz, J.A., (2004) A molecular link between SR protein dephosphorylation and mRNA export. Proc Natl Acad Sci U S A 101, 9666-9670.

Jimeno, S., Rondon, A.G., Luna, R., Aguilera, A., (2002) The yeast THO complex and mRNA export factors link RNA metabolism with transcription and genome instability. Embo J 21, 3526-3535. 
Katahira, J., Inoue, H., Hurt, E., Yoneda, Y., (2009) Adaptor Aly and co-adaptor Thoc5 function in the Tap-p15-mediated nuclear export of HSP70 mRNA. Embo J 28, $556-567$.

Katahira, J., Strasser, K., Podtelejnikov, A., Mann, M., Jung, J.U., Hurt, E., (1999) The Mex67p-mediated nuclear mRNA export pathway is conserved from yeast to human. Embo J 18, 2593-2609.

Kohler, A., Hurt, E., (2007) Exporting RNA from the nucleus to the cytoplasm. Nat Rev Mol Cell Biol 8, 761-773.

Komili, S., Silver, P.A., (2008) Coupling and coordination in gene expression processes: a systems biology view. Nat Rev Genet 9, 38-48.

Laimins, L.A., Tsichlis, P., Khoury, G., (1984) Multiple enhancer domains in the $3^{\prime}$ terminus of the Prague strain of Rous sarcoma virus. Nucleic Acids Res 12, 6427-6442.

Lee, C.L., Pang, P.C., Yeung, W.S., Tissot, B., Panico, M., Lao, T.T., Chu, I.K., Lee, K.F., Chung, M.K., Lam, K.K., Koistinen, R., Koistinen, H., Seppala, M., Morris, H.R., Dell, A., Chiu, P.C., (2009) Effects of differential glycosylation of glycodelins on lymphocyte survival. J Biol Chem 284, 15084-15096.

Li, H., d'Anjou, M., (2009) Pharmacological significance of glycosylation in therapeutic proteins. Curr Opin Biotechnol 20, 678-684. 
Maniatis, T., Reed, R., (2002) An extensive network of coupling among gene expression machines. Nature 416, 499-506.

Masuda, S., Das, R., Cheng, H., Hurt, E., Dorman, N., Reed, R., (2005) Recruitment of the human TREX complex to mRNA during splicing. Genes Dev 19, 1512-1517.

Masuda, S., Kobayashi, T., Chikuma, M., Nagao, M., Sasaki, R., (2000a) The oviduct produces erythropoietin in an estrogen- and oxygen-dependent manner. Am J Physiol Endocrinol Metab 278, E1038-1044.

Masuda, S., Moon, S.K., Kambe, T., Nagao, M., Sasaki, R., (2000b) A new biological strategy for high productivity of recombinant proteins in animal cells by the use of hypoxia-response enhancer. Biotechnol Bioeng 67, 157-164.

Masuda, S., Nagao, M., Sasaki, R., (1999) Erythropoietic, neurotrophic, and angiogenic functions of erythropoietin and regulation of erythropoietin production. Int J Hematol $70,1-6$.

Masuda, S., Okano, M., Yamagishi, K., Nagao, M., Ueda, M., Sasaki, R., (1994) A novel site of erythropoietin production. Oxygen-dependent production in cultured rat astrocytes. J Biol Chem 269, 19488-19493.

Mizushima, S., Nagata, S., (1990) pEF-BOS, a powerful mammalian expression vector. Nucleic Acids Res 18, 5322. 
Mohan, C., Kim, Y.G., Koo, J., Lee, G.M., (2008) Assessment of cell engineering strategies for improved therapeutic protein production in $\mathrm{CHO}$ cells. Biotechnol J 3, 624-630.

Moraes, K.C., (2010) RNA surveillance: molecular approaches in transcript quality control and their implications in clinical diseases. Mol Med 16, 53-68.

Nagao, M., Masuda, S., Abe, S., Ueda, M., Sasaki, R., (1992) Production and ligand-binding characteristics of the soluble form of murine erythropoietin receptor. Biochem Biophys Res Commun 188, 888-897.

Okano, M., Suga, H., Masuda, S., Nagao, M., Narita, H., Ikura, K., Sasaki, R., (1993) Characterization of erythropoietin isolated from rat serum: biochemical comparison of rat and human erythropoietins. Biosci Biotechnol Biochem 57, 1882-1885.

Orphanides, G., Reinberg, D., (2002) A unified theory of gene expression. Cell 108, $439-451$.

Padler-Karavani, V., Yu, H., Cao, H., Chokhawala, H., Karp, F., Varki, N., Chen, X., Varki, A., (2008) Diversity in specificity, abundance, and composition of anti-Neu5Gc antibodies in normal humans: potential implications for disease. Glycobiology 18, 818-830.

Reed, R., Cheng, H., (2005) TREX, SR proteins and export of mRNA. Curr Opin Cell Biol 17, 269-273. 
Reed, R., Hurt, E., (2002) A conserved mRNA export machinery coupled to pre-mRNA splicing. Cell 108, 523-531.

Sauer, B., (1994) Site-specific recombination: developments and applications. Curr Opin Biotechnol 5, 521-527.

Seppala, M., Koistinen, H., Koistinen, R., Chiu, P.C., Yeung, W.S., (2007)

Glycosylation related actions of glycodelin: gamete, cumulus cell, immune cell and clinical associations. Hum Reprod Update 13, 275-287.

Sethuraman, N., Stadheim, T.A., (2006) Challenges in therapeutic glycoprotein production. Curr Opin Biotechnol 17, 341-346.

Stenberg, R.M., Thomsen, D.R., Stinski, M.F., (1984) Structural analysis of the major immediate early gene of human cytomegalovirus. J Virol 49, 190-199.

Stinski, M.F., Roehr, T.J., (1985) Activation of the major immediate early gene of human cytomegalovirus by cis-acting elements in the promoter-regulatory sequence and by virus-specific trans-acting components. J Virol 55, 431-441.

Strasser, K., Masuda, S., Mason, P., Pfannstiel, J., Oppizzi, M., Rodriguez-Navarro, S., Rondon, A.G., Aguilera, A., Struhl, K., Reed, R., Hurt, E., (2002) TREX is a conserved complex coupling transcription with messenger RNA export. Nature 417, 304-308. 
Stutz, F., Izaurralde, E., (2003) The interplay of nuclear mRNP assembly, mRNA surveillance and export. Trends Cell Biol 13, 319-327.

Suttie, J.W., (1986) Report of Workshop on expression of vitamin K-dependent proteins in bacterial and mammalian cells, Madison, Wisconsin, USA, April 1986. Thromb Res 44, 129-134.

Suzuki, T., Ishihara, K., Migaki, H., Ishihara, K., Nagao, M., Yamaguchi-Iwai, Y., Kambe, T., (2005) Two different zinc transport complexes of cation diffusion facilitator proteins localized in the secretory pathway operate to activate alkaline phosphatases in vertebrate cells. J Biol Chem 280, 30956-30962.

Tabernero, C., Zolotukhin, A.S., Valentin, A., Pavlakis, G.N., Felber, B.K., (1996) The posttranscriptional control element of the simian retrovirus type 1 forms an extensive RNA secondary structure necessary for its function. J Virol 70, 5998-6011.

Tangvoranuntakul, P., Gagneux, P., Diaz, S., Bardor, M., Varki, N., Varki, A., Muchmore, E., (2003) Human uptake and incorporation of an immunogenic nonhuman dietary sialic acid. Proc Natl Acad Sci U S A 100, 12045-12050.

Vinciguerra, P., Stutz, F., (2004) mRNA export: an assembly line from genes to nuclear pores. Curr Opin Cell Biol 16, 285-292.

Wakabayashi-Ito, N., Nagata, S., (1994) Characterization of the regulatory elements in the promoter of the human elongation factor-1 alpha gene. J Biol Chem 269, 
29831-29837.

Yamamoto, T., de Crombrugghe, B., Pastan, I., (1980) Identification of a functional promoter in the long terminal repeat of Rous sarcoma virus. Cell 22, 787-797.

Yamazaki, T., Fujiwara, N., Yukinaga, H., Ebisuya, M., Shiki, T., Kurihara, T., Kioka, N., Kambe, T., Nagao, M., Nishida, E., Masuda, S., (2010) The closely related RNA helicases, UAP56 and URH49, preferentially form distinct mRNA export machineries and coordinately regulate mitotic progression. Mol Biol Cell 21, 2953-2965.

Zhou, Z., Luo, M.J., Straesser, K., Katahira, J., Hurt, E., Reed, R., (2000) The protein Aly links pre-messenger-RNA splicing to nuclear export in metazoans. Nature 407, 401-405.

Zolotukhin, A.S., Michalowski, D., Smulevitch, S., Felber, B.K., (2001) Retroviral constitutive transport element evolved from cellular TAP(NXF1)-binding sequences. J Virol 75, 5567-5575.

Zolotukhin, A.S., Valentin, A., Pavlakis, G.N., Felber, B.K., (1994) Continuous propagation of $\operatorname{REE}(-)$ and $\operatorname{Rev}(-) \operatorname{RE}(-)$ human immunodeficiency virus type 1 molecular clones containing a cis-acting element of simian retrovirus type 1 in human peripheral blood lymphocytes. J Virol 68, 7944-7952. 


\section{Figure legends}

Fig. 1. The presence of a constitutive transport element (CTE) enhanced the expression of luciferase in four human cell lines.

A, HeLa cells; B, MCF7 cells; C, U2OS cells; D, A549 cells. CTE(-) and CTE(+) indicate the absence or presence of a CTE in the expression plasmid, respectively. Luciferase activity was normalized to $\beta$-galactosidase and is indicated by fold induction. The data shown are the means \pm SD of triplicate experiments.

Fig. 2. The presence of a constitutive transport element (CTE) and Tap-p15 individually and synergistically enhanced the expression of reporter proteins in HeLa cells.

A, C, E, Luciferase (Luc), secreted alkaline phosphatase (SEAP) and erythropoietin (EPO) expressions, respectively, were measured and normalized to total protein content. The data shown are the means \pm SD of triplicate experiments. $\mathrm{B}, \mathrm{D}, \mathrm{F}$, western blotting was performed using anti-HA, anti-Flag and anti-actin antibodies to detect HA-Tap, Flag-p15 and endogenous actin, respectively, for normalization.

Fig. 3. The presence of a constitutive transport element (CTE) enhanced the expression of erythropoietin (EPO) in Flpin293Trex stably transfected cell lines. CTE(-) and $\mathrm{CTE}(+)$ indicate the absence or presence of a CTE in the expression plasmid, respectively. A, EPO secretion in the culture media was normalized to total protein content and is indicated as fold induction. The data shown are the means \pm SD of triplicate experiments. B, The western analysis of EPO protein. EPO secretion in the culture media was detected with anti-EPO polyclonal antibody. C, The EPO production with or without CTE element was measured by sandwich enzyme-linked immunosorbent assay. The EPO production in the absence and presence of CTE was 
indicated with closed circle and closed square, respectively. The data shown are the means \pm SD of triplicate experiments.

Fig. 4. The expression of RNA-binding proteins enhanced erythropoietin (EPO) production in Flpin293Trex stably transfected cell lines.

A, C, EPO secretion in the culture media was measured and normalized to total protein content. The data shown are the means \pm SD of triplicate experiments. $\mathrm{B}, \mathrm{D}$, Western blotting was performed using anti-Flag, anti-HA and anti-actin antibodies to detect HA-Tap, Flag-fusion proteins of 9G8, p15, SF2 and Aly, and endogenous actin, respectively, for normalization. The bands for 9G8 and SF2 are broad because of the different levels of serine phosphorylation in the RS domain of SR proteins.

\section{Supplemental Figure legends}

Fig. 1. Strategy for efficient recombinant protein expression using a viral constitutive transport element (CTE) and mRNA-binding proteins.

A, mRNA maturation and export in mammalian cells. In mammalian cells, most pre-mRNAs contain introns, which are removed by mRNA splicing. Some genes are intronless. The hTREX complex is recruited onto both types of mRNA. Aly is a component of the hTREX complex and recruits Tap onto mRNA. The SR proteins 9G8 and SF2 are recruited onto mRNA during splicing and recruit Tap onto mRNA.

Tap-p15 is thus recruited onto mRNA through its association with Aly or SR proteins. Viral RNAs containing a CTE sequence directly associate with Tap. The heterodimer Tap-p15 functions as an mRNA export receptor. (AAAAA)n indicates 3' 
polyadenylation of the mRNA. B, Schematics of the plasmids used. SV40P, SV40 promoter; CMVP, CMV promoter; Luc, luciferase; SEAP, secreted alkaline phosphatase; EPO, erythropoietin; CTE, constitutive transport element.

Fig. 2. The presence of a constitutive transport element (CTE) did not enhance the expression of luciferase in $\mathrm{CHO}$ and $\mathrm{BHK}$ cell lines. A, CHO cells; B, BHK cells; (-) and (+) indicate the absence or presence of a CTE in the expression plasmid, respectively. Luciferase activity was normalized to $\beta$-galactosidase and is indicated by fold induction. The data shown are the means \pm SD of triplicate experiments. $\mathrm{C}$, Luciferase activity was normalized to total protein content and is indicated as fold induction. The data shown are the means \pm SD of triplicate experiments. D, Western blotting was performed using anti-HA, anti-Flag and anti-actin antibodies to detect HA-Tap, Flag-p15 and endogenous actin, respectively, for normalization. 

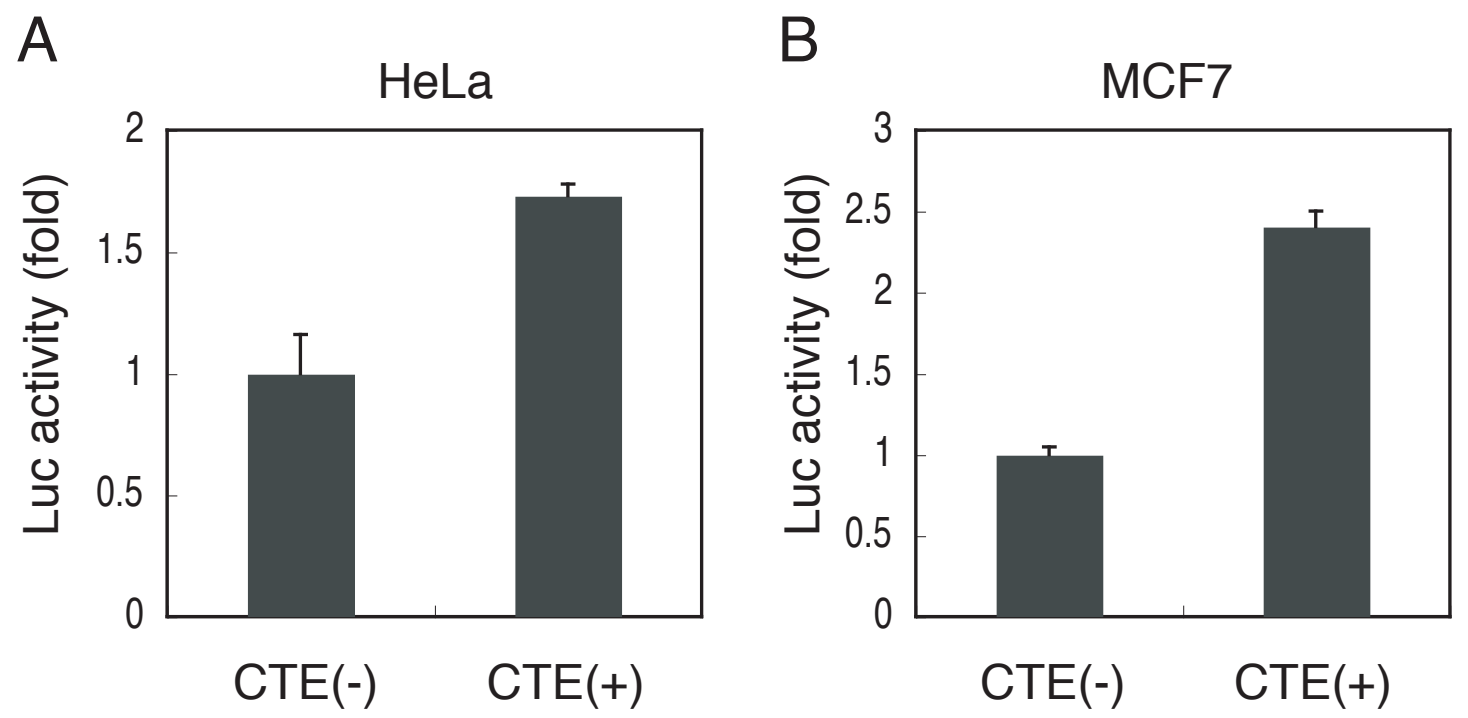

C
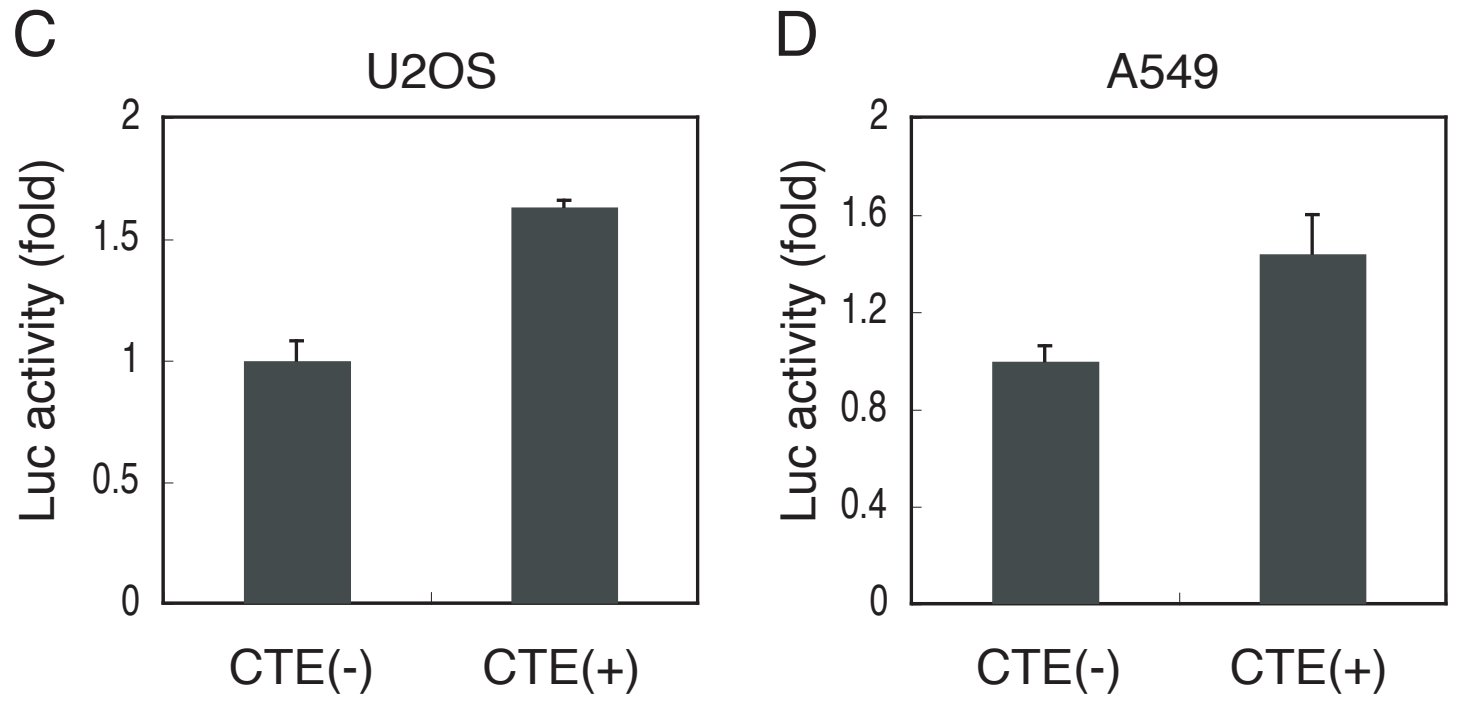

Fig. 1 


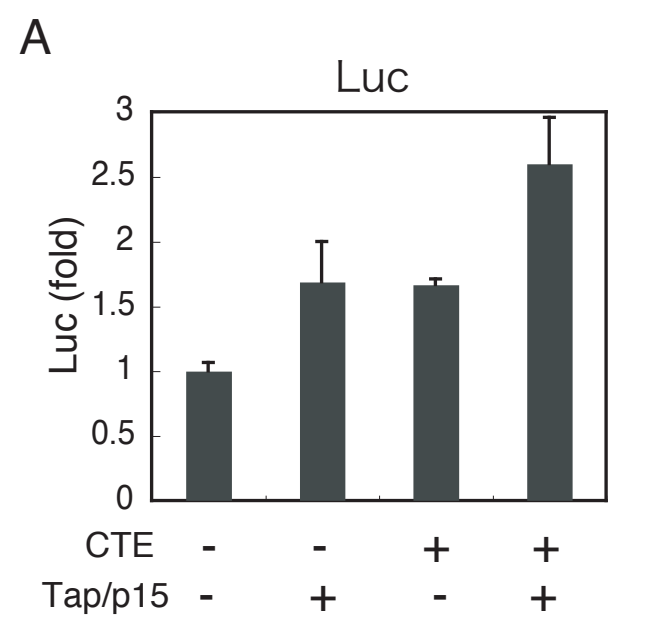

C

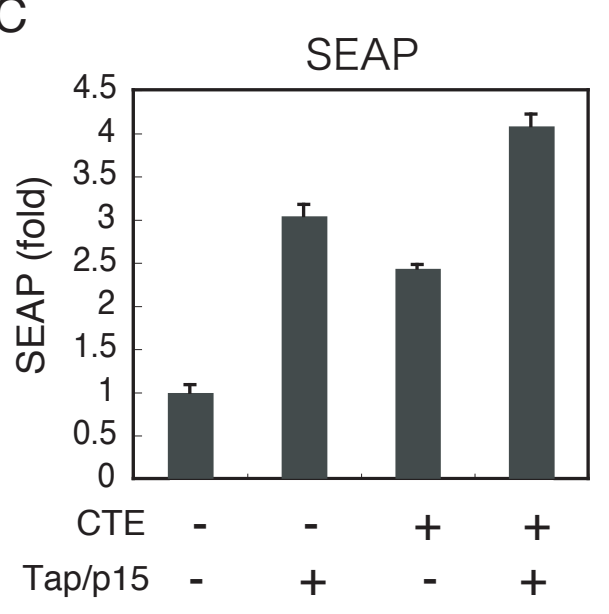

E

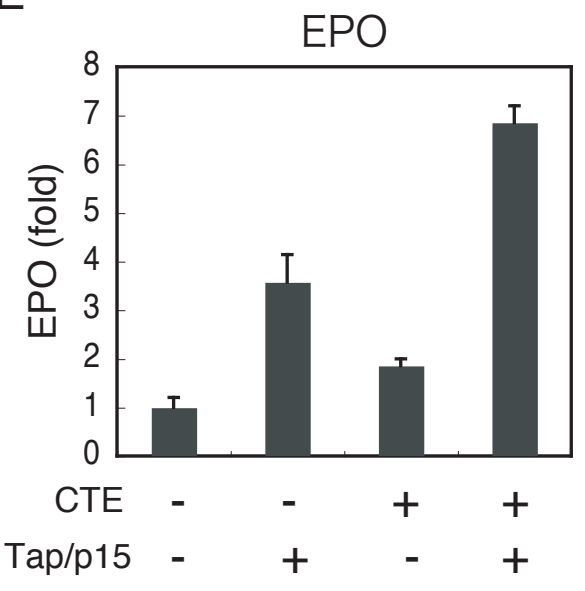

B

D

WB:

Luc

HA-Tap

WB:

SEAP

HA-Tap

WB:

F

Flag-p15

Flag-p15

Actin

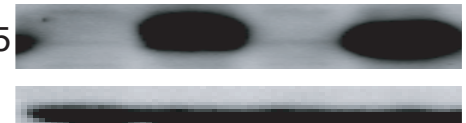

HA-Tap

Flag-p15

Actin

$\begin{array}{rrrrr}\text { CTE } & - & - & + & + \\ \text { Tap/p15 } & - & + & - & +\end{array}$

CTE - $\quad-\quad$ - $\quad+\quad+$

Tap/p15 - $\quad+\quad-\quad+$

Fig. 2 
A

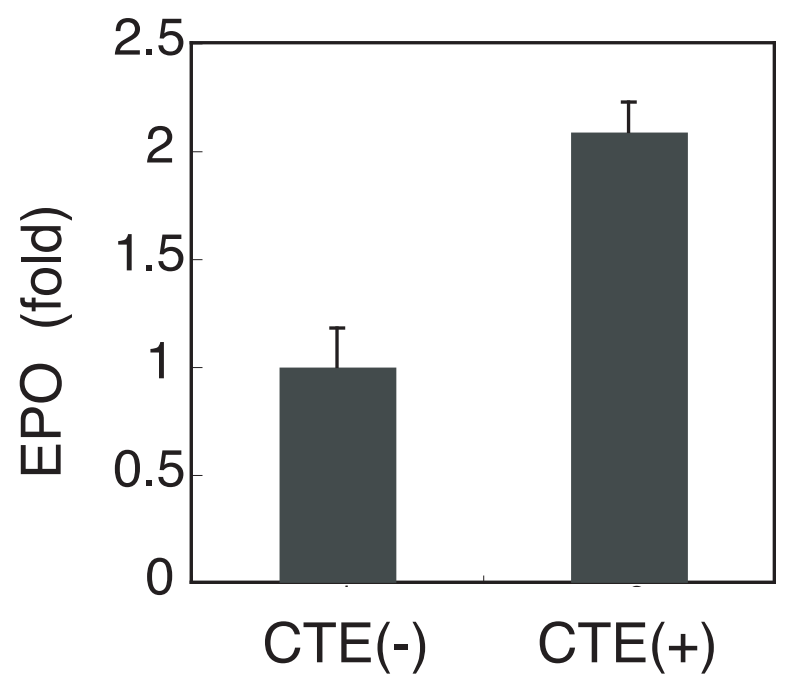

B

CTE(-) CTE $(+)$

$\mathrm{kDa}$

$35-$

$25-$

C

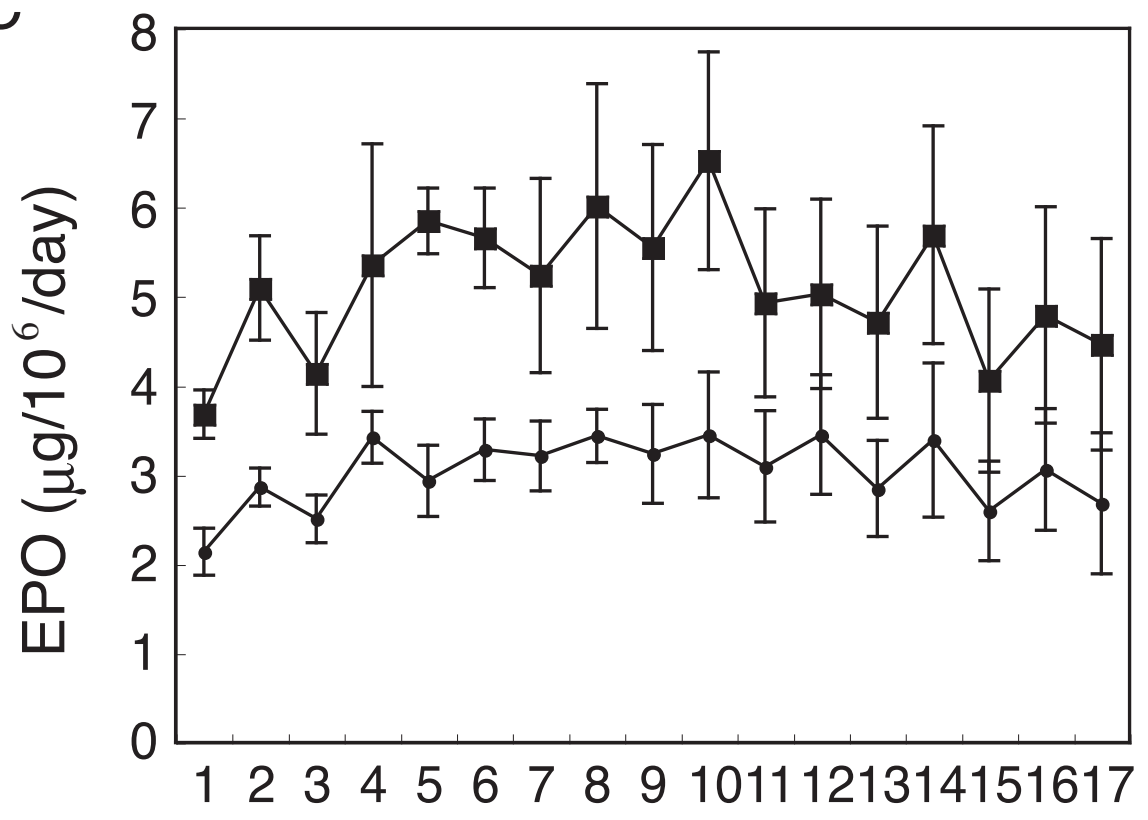

Culture day

Fig. 3 
A

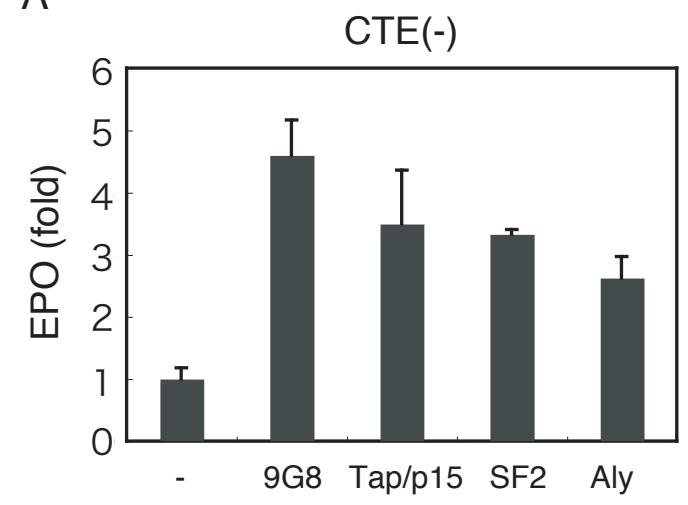

B

CTE(-)

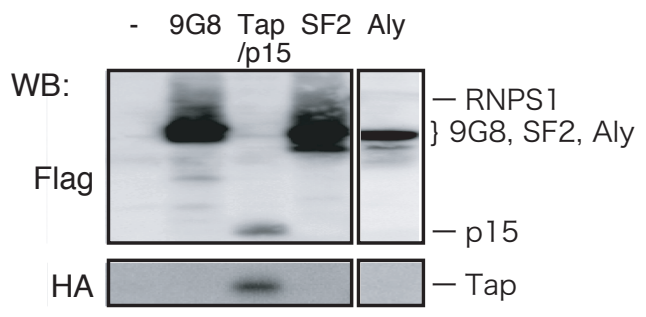

Actin
C

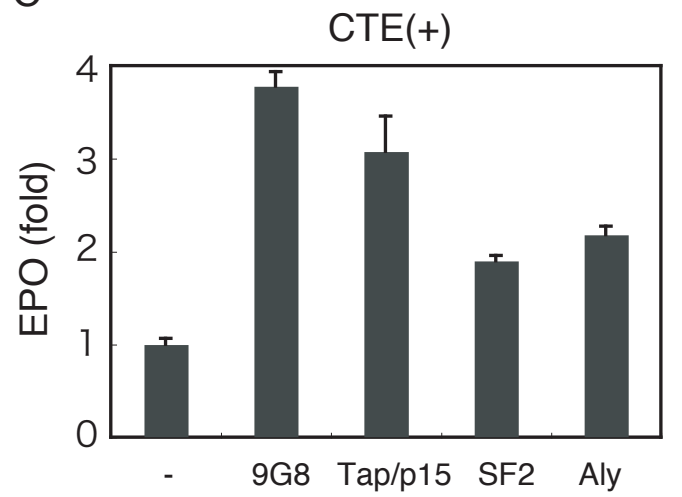

D

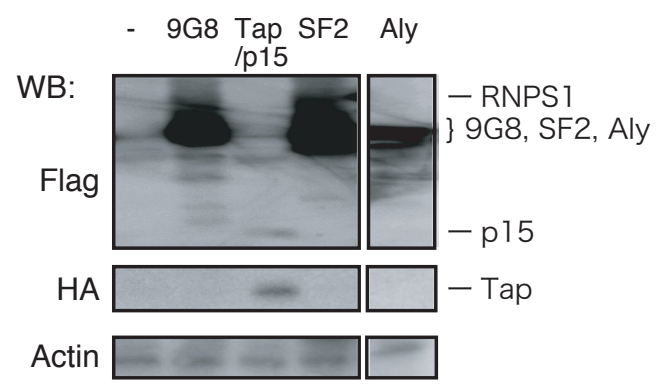

Fig. 4 
A

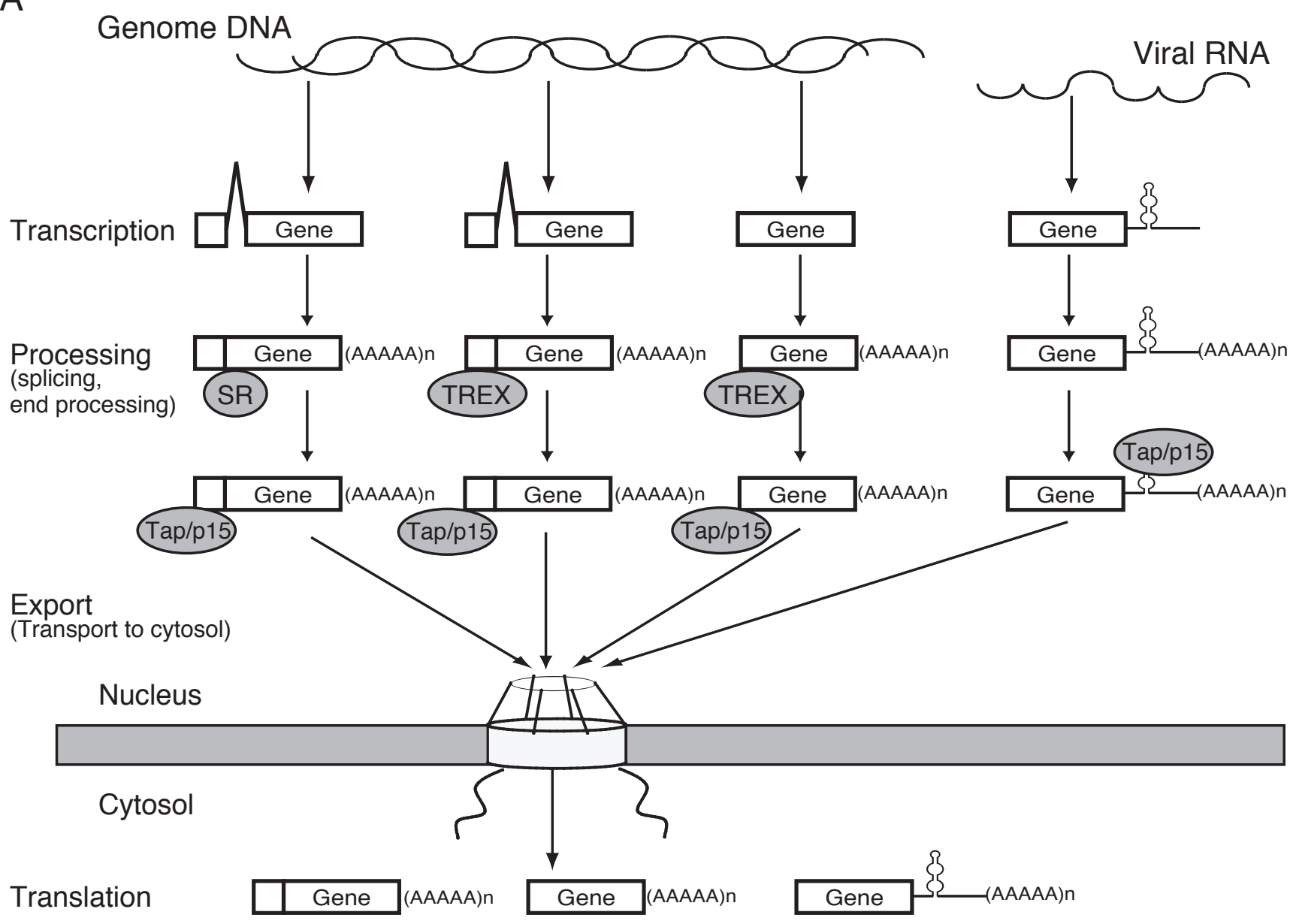

Aly) THO complex

TREX:

B
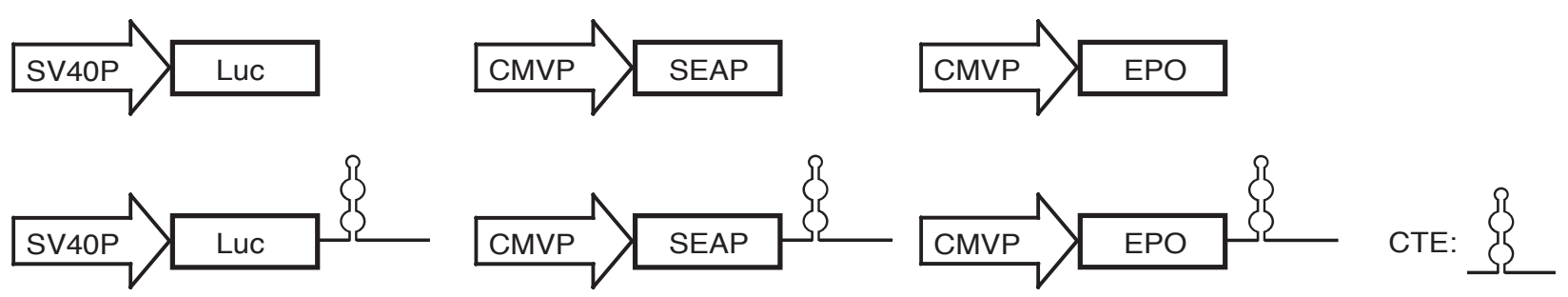
A

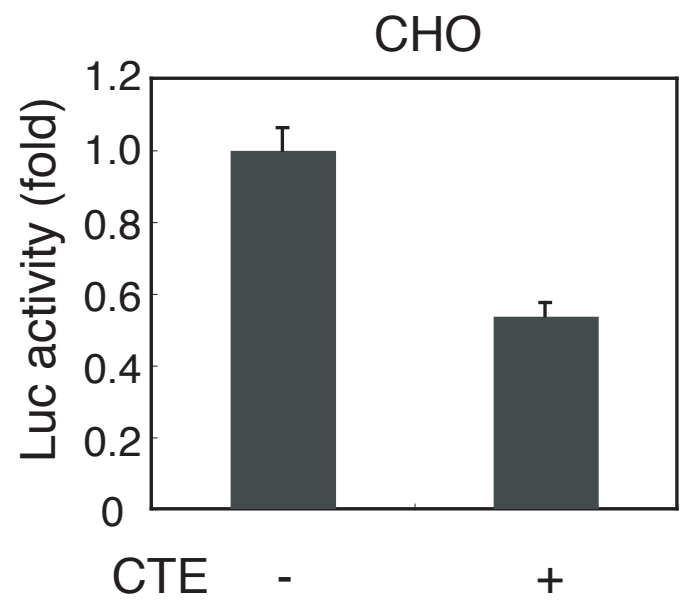

B

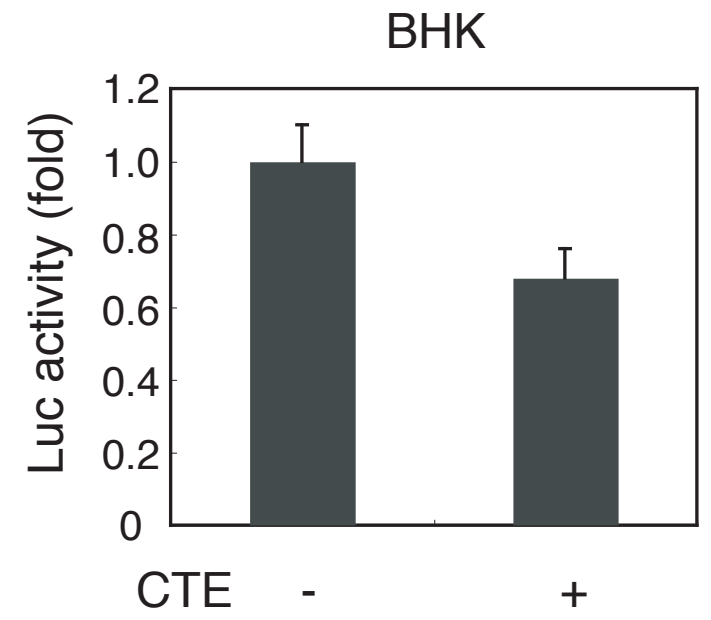

C

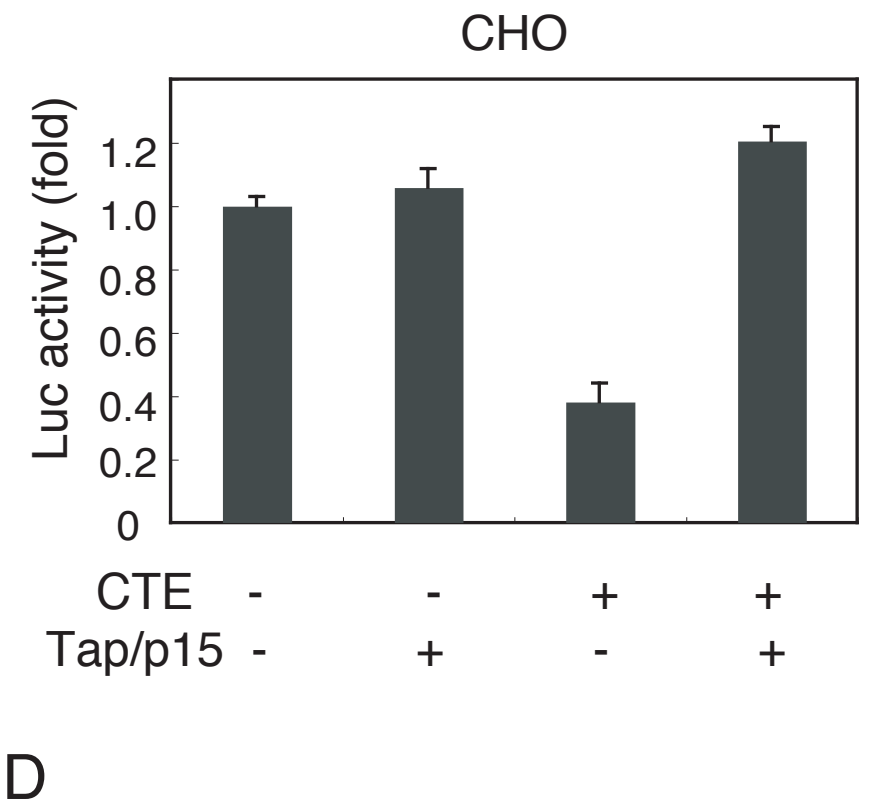

WB:

HA-Tap

Flag-p15

Actin

CTE - $\quad-\quad+\quad+$

Tap/p15 - + + + 